\title{
A Smart Substation Field Secondary Device Testing Technique Based on Recurrence Principle
}

\author{
Kanjun Zhang ${ }^{*}$, Lei Chen ${ }^{2}$, Yongjun Xia ${ }^{1}$, Yang Lei ${ }^{1}$, Xin Shu ${ }^{1}$, Hengxuan Li ${ }^{1}$, Ting Wang1, \\ Zhenan Du' \\ ${ }^{1}$ State Grid Hubei Electric Power Research Institute, Wuhan, China \\ ${ }^{2}$ Wuhan Fangyuan Dongli Electric Power Technology Center, Wuhan, China \\ Email: ${ }^{*}$ zhangzkj7779@163.com
}

Received December 2013

\begin{abstract}
According to the demand of substation secondary device dynamic performance testing, a smart substation field testing technique based on recurrence principle is proposed in the paper, and the characteristics of smart substation secondary device digitization and information sharing are used by the technique. The principle of testing technique is as follow: the digital simulation model is constructed on the basis of the substation's actual construction, then the simulating data highly similar to substation's actual electric quantity transient process is generated, at last, the substation digital secondary device can be tested by using data "recurrence" technique. The testing technique is verified and applied by constructing testing system, the application results show that the technique can effectively perform field test on the dynamic performance of digital secondary device, and the technique has good engineering implementation and application value.
\end{abstract}

\section{Keywords}

Smart Substation; Secondary Device; Dynamic Performance; Data Recurrence; Field Testing Technique

\section{Introduction}

In conventional substation, the performance of secondary equipments (e.g. relay protection and automatic devices) is usually tested by using protective relay test device, but the method is only for secondary equipments' static performance, and in some cases (e.g. fault analysis), the dynamic performance of secondary equipment needs to be tested [1,2]. Using digital-analogical hybrid simulation system (e.g. RTDS, ADPSS) can test the dynamic performance of secondary equipment effectively [3,4], but the test can only be carried out in laboratory, the test period is usually long, and the number of tested equipments is also limited, in short the method cannot perfectly fit the requirements of actual operation. Using portable testing simulation devices is another method,

*Corresponding author.

How to cite this paper: Zhang, K.J., Chen, L., Xia, Y.J., Lei, Y., Shu, X., Li, H.X., Wang, T. and Du, Z.N. (2014) A Smart Substation Field Secondary Device Testing Technique Based on Recurrence Principle. Journal of Power and Energy Engineering, 2, 244-251. http://dx.doi.org/10.4236/ipee.2014.24035 
the device contains digital simulation models, can output voltage, current and switch signals which can test the dynamic performance of secondary equipments [5,6], moreover the device can be carried to substation feasibly. However, the portable testing device can only store certain fixed simulation model which may be different from the actual structure of power grid, the limitation lead to negative effectiveness of dynamic tests, so how to test the dynamic performance of secondary equipments according to engineering need is a technical issue to be resolved.

Although the field testing methods and modes of secondary equipment in smart substation are not essentially different from that of conventional substation, the signal transmission modes of secondary equipment in smart substation are vary from conventional substation extremely [7-9]. So based on the features such as secondary equipment digitalization and information sharing in smart substation, testing the secondary equipment's dynamic performance in substation can be done.

On the basis of above idea, a new field testing technology of secondary equipment in smart substation is presented in the paper, and its validity will be verified by corresponding test system.

\section{Field Dynamic Testing Analysis of Secondary Equipment in Smart Substation}

In conventional substation, the signals inputted to secondary equipment are electrical and switch (Figure 1(a)). During dynamic performance testing, the change of input signals' value and phase may very complex in order to comply with power grid transient process, in this case the manual operation is difficult to simulate effectively. But because of the change of signal transmission modes in smart substation, manual input dynamic signal during the test becomes possible. At present, the relay protection and automatic devices in smart substation generally use the "point-to-point sampling and tripping" method for transmissing signals [10], the process is shown in Figure 1(b).

In Figure 1, whether the transformers used in smart substation are conventional or electronic, the signals inputted to relay protection and automatic devices are all digital message. So if the dynamic test signals are changed into corresponding digital messages (SV, GOOSE message), and input the messages to the secondary equipments, then dynamic testing can be done.

In total, the conditions of secondary equipments dynamic performance field testing in smart substation are as follows:

1) Similar to actual operating conditions of substation. The output test signals should simulate the corresponding operating conditions in substation, that is to say, the transient process of test signals should be similar to that of actual electrical and switch quantities.

2) The format of test signals complies with the requirement of digital secondary equipment. Test signals' format complies with IEC61850 standard digital message, and the signals can be directly used to test.

3) Test signals can be carried or transmitted to substation to test secondary equipments.

4) Test signals have synchronic output function. As to transformer protection and bus differential protection which may require signal outputted from more than one test equipment, the output signal of each test equipment should be synchronized.

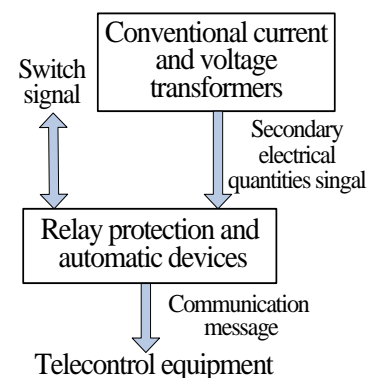

(a)

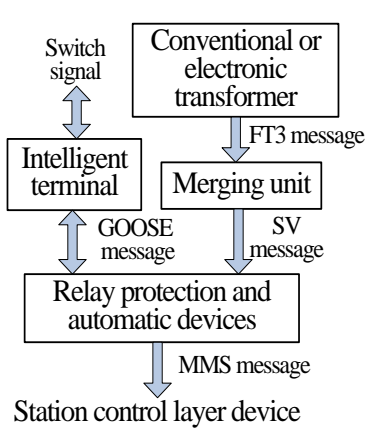

(b)

Figure 1. Schematic diagram of substation secondary device signal transmission comparison. (a) Conventional substation; (b) Smart substation. 


\section{New Field Test Technology of Secondary Equipment in Smart Substation}

\subsection{Principle of New Test Technology}

The new field test technology of smart substation secondary equipment (new test technology in short) proposed in the paper is composed of five parts, they are generating, conversion, storage, output, and synchronization of the dynamic signals. The specific principle is: Step 1, generate dynamic test signals by simulating substation's operating conditions, and the transient process of signals is similar to the actual electrical quantities. Step 2, convert the signals to data messages which meet requirements of IEC61850 protocol. Step 3, store the messages in test devices. Step 4, test the dynamic performance of digital secondary equipment by output the messages in substation. And during the test, the test signals outputted by multiple test devices can be synchronized by coordination of clock signal.

\subsection{Implementation of New Test Technology}

The implementation principle of new test technology is shown in Figure 2.

The test system in Figure 2 is composed of five parts: signal source unit, signal converting unit, signal storage unit, test output unit and signal synchronizing unit. The specific implementation scheme of each part is as follows.

\subsubsection{Signal Source Unit}

Constitute the signal source unit by using digital simulation system. The implementation procedure is as follows: Firstly, establish the corresponding digital simulation model of power grid and substations, Secondly, calculate by electrical-electromagnetic transient hybrid simulation mode [11-13]. Specifically, the model of substations and nearby power grid is used by electromagnetic transient simulation model, the other part of power grid uses electromechanical transient simulation model. The above technical can reduce the negative influence of model simplifying on the calculation results accuracy. At last, produce the simulation results which are similar to the actual electrical quantities by simulating various operating conditions of substation.

\subsubsection{Signal Conversion Unit}

Convert the output signals to messages which meet the requirements of IEC61850 protocol, there are two conversion methods can be chosen.

1) Method 1: convert the output signals directly, this conversion method requires less links, but need to develop a special signal conversion equipment.

2) Method 2: convert the output signals to normal secondary electrical and switch quantities by using physical interface devices and power amplifiers, and then turn the quantities to data messages which meet the requirements of IEC61850 protocol by merging units and smart terminal. This conversion method has more links, but more mature and easy to imply.

Method 2 is chosen based on above analysis.

\subsubsection{Signal Storage and Output Unit}

Recurrence output the data messages in substation, reproduce the electrical quantities' transient process during actual operating condition of substation, and test the secondary equipment. The "recurrence" output of messages

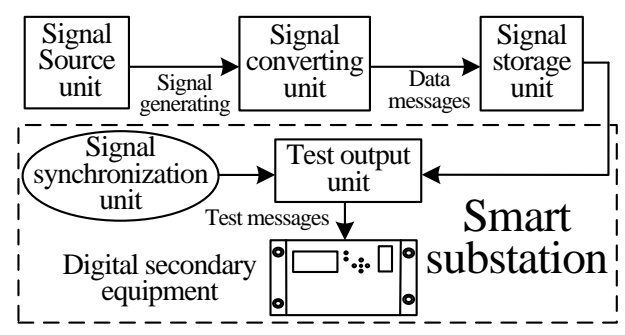

Figure 2. Principle diagram of new testing technique. 
can be achieved in two ways:

1) Convert the data messages to recorded data (COMTRADE format), store the data into digital relay test device. And then convert the recorded data to IEC61850-9-2 data messages by using the recorded data recurrence function of digital relay test device.

2) Recurrence output the data messages after edit. This method does not need conversion, but need to develop a special data processing software.

The first method is used because it is easy to implement.

\subsubsection{Signal Synchronizing Unit}

When carry out test of the secondary equipments which need multi-interval messages, single test equipment may not meet the requirements. Then multiple test devices will be used, and the test signals outputted by these test devices can be synchronized by the alignment time signal which is outputted by signal synchronization unit. The alignment time mode such as IRIG-B or IEEE1588 can be used [14,15].

\subsection{Test System}

Building test system which is shown in Figure 3.

In Figure 3, the signal source unit is consisted of advanced digital power system simulator (ADPSS) and physical interface device (PID-B1). Signal conversion unit includes power amplifier (PW60A), merging unit (MU) and smart terminal (ST). Signal storage unit contains network analyzer (ZH-5N) and portable computers (PC). Output unit is digital relay protection device (PNF801). Synchronization unit uses clock synchronization device (ZH-502).

\subsection{Validation of New Test Technology}

The test process has multiple links, so validity of signals transmitting needs to be verified. In addition, the synchronization of signals outputted from different test equipment needs also to be verified. The test model is shown in Figure 4. The main component of model is a three winding transformer, the test system has three intervals, it includes three Merging units, electronic current and voltage transformers (ECT, EVT), three sets of digital relay test device are used.

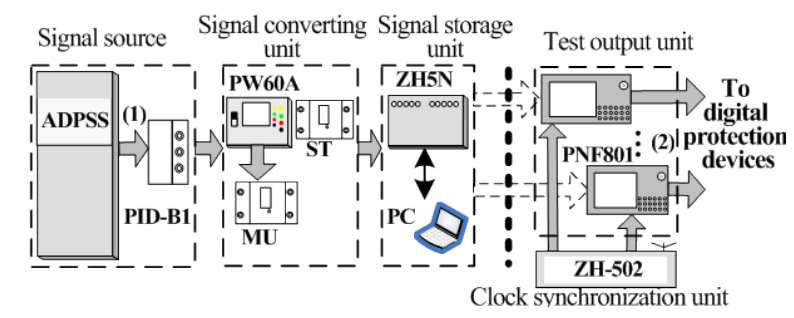

Figure 3. Schematic diagram of test system structure.

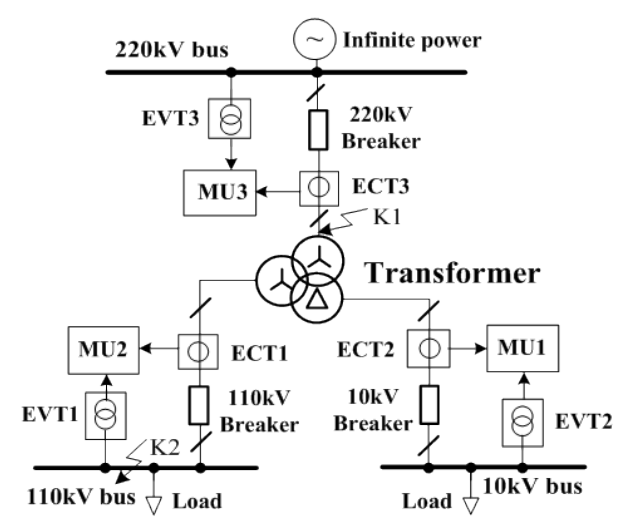

Figure 4. Schematic diagram of testing model 1. 
1) The Validity of Signal Recurrence

Take phase A ground fault (Figure 4, K1) of transformer $220 \mathrm{kV}$ side for example, compare the output signals of ADPSS and test output unit (Figure 3, link (1) and (2)), then the accuracy of recurrence data can be checked. The comparison results are shown in Table 1 and Figure 5.

In Figure 5, red dashed and solid black lines represent the output data of digital relay test device and ADPSS respectively. From the comparison results in Table 1 and Figure 5, the error is very low, then the validity of recurrence output signal results is verified.

2) Verification of Signal Synchronization

Take phase A and B metallic short circuit fault (Figure 4, K2) of transformer $110 \mathrm{kV}$ side for example to test the synchronization of output signals, IRIG-B method is used, the transformer differential current around the fault time is shown in Figure 6.

Because K2 is located out of transformer differential protection zone, the differential current is approximately zero when the output signals of all digital relay test devices are synchronous. The differential current of three phases in Figure 6 is less than $2.4 \times 10^{-3} \mathrm{I}_{\mathrm{e}}$ (transformer rated current), so the synchronization of test messages is verified.

\section{The Application of New Test Technology}

Testing the transformer protection device of a $220 \mathrm{kV}$ smart substation with the new test technology. Building the test model according to the actual structure of substation, the model and associated test equipment is shown in Figure 7.

In Figure 7, the test model includes $220 \mathrm{kV}$ double bus bar, a three winding transformer and a $220 \mathrm{kV}$ transmission line. So test system has seven intervals, it contains seven combined unit (MU1-MU7), six sets of intelligent terminals (ST1-ST6), six sets of electronic current transformer (ECT1-ECT6), electronic voltage transformer (ETV1-ETV6) and corresponding collector. The output unit consists of seven same type digital relay test devices. K1-K5 is short circuit fault point.

Table 1. Comparison of transformer phase A current value.

\begin{tabular}{cccccccc}
\hline \multirow{2}{*}{ No. } & Measuring points & \multicolumn{3}{c}{ Pre-fault } & \multicolumn{3}{c}{ Post-fault } \\
\cline { 3 - 7 } & & Simulation & Recurrence & Error & Simulation & Recurrence & Error \\
\hline 1 & $220 \mathrm{kV}$ Side Current (A) & 380.7 & 380.1 & $0.17 \%$ & 3323.1 & 3321.4 & $0.05 \%$ \\
2 & $110 \mathrm{kV}$ Side Current (A) & 575.4 & 574.9 & $0.09 \%$ & 557.6 & 557.4 & $0.03 \%$ \\
3 & $10 \mathrm{kV}$ Side Current (A) & 1941.0 & 1941.4 & $0.02 \%$ & 1846.9 & 1846.1 \\
\hline
\end{tabular}

Tag: The current in Table 1 is RMS of one cycle pre-fault and post-fault.
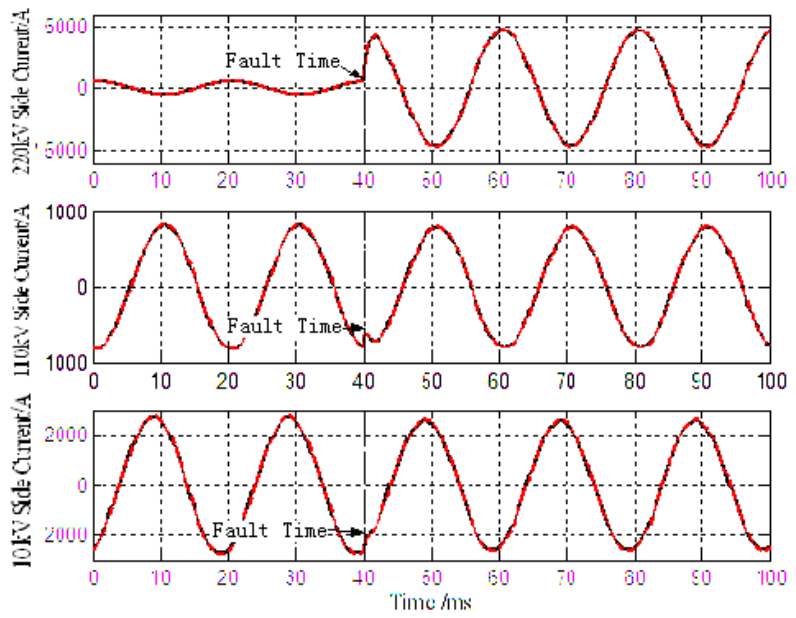

Figure 5. Comparison of phase A current waveform. 


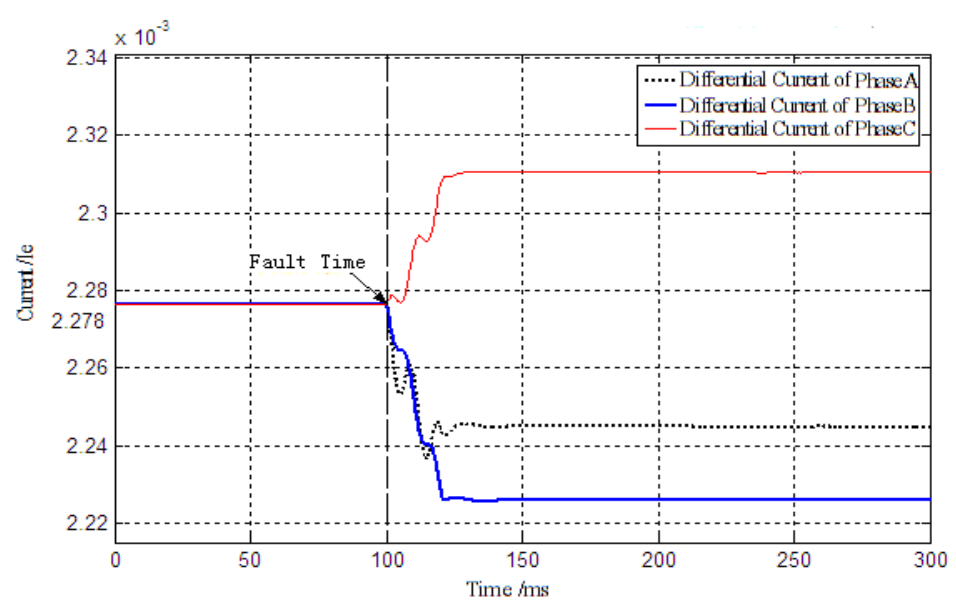

Figure 6. Effective value of transformer differential current.

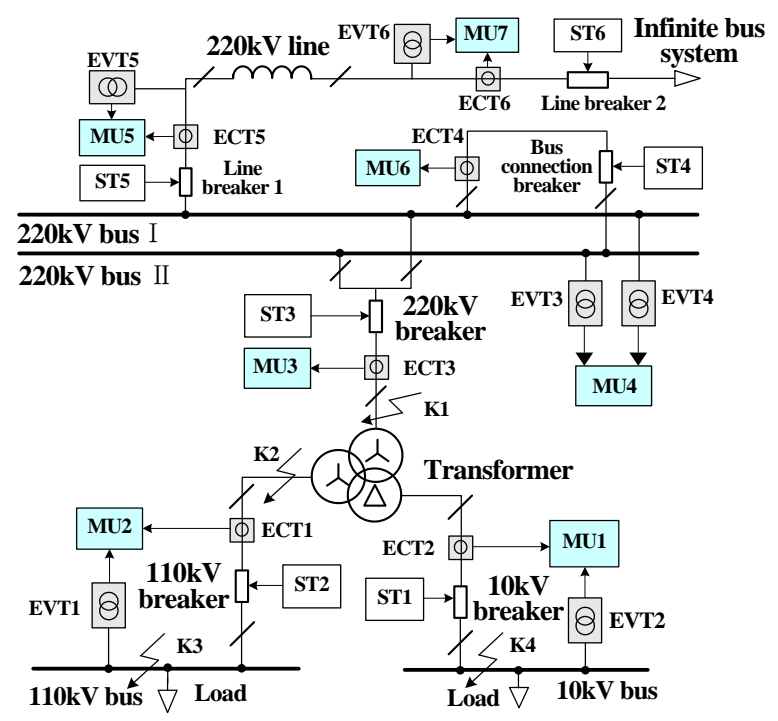

Figure 7. Schematic diagram of testing model 2.

The test items include: metallic short circuit which occurs in and out of transformer differential protection zone, transition resistance short circuit, transitional fault, power grid oscillation and short circuit during oscillation etc. The last three types in above faults can better test the dynamic performance of secondary equipment, the testing results are shown in Figures 8 and 9.

In Figures 8 and 9, $U_{A}, U_{B}$ and $U_{C}$ are three-phase voltage of $220 \mathrm{kV}$ bus bar, $I_{A}, I_{B}$ and $I_{C}$ are three-phase current of transformer $220 \mathrm{kV}$ side, T1 is protection starting signal (switch signal), T2 is protection operating signal, the starting time is $0 \mathrm{~ms}$.

In Figure 8, during power grid oscillation, phase A ground fault occurred on $110 \mathrm{kV}$ bus bar (Figure 7, K3, outside of transformer differential protection areas) is simulated. $507 \mathrm{~ms}$ after the fault occurring, the multiple voltage over-current protection segment I of transformer $110 \mathrm{kV}$ side operates (protection delay setting is 500 $\mathrm{ms}$ ), and the breakers of transformer three sides trip, other protections have not operated.

In Figure 9, a transitional fault is simulated. At first, Phase A ground fault (Figure 7 K3) occurs during power grid oscillation, and then after $60 \mathrm{~ms}$, phase B (Figure $7 \mathrm{~K} 2$, inside of transformer differential protection areas) occurs ground fault. During the test, $79 \mathrm{~ms}$ after the protection start (T1), the transformer ratio differential protection operates, and the breakers of transformer three sides trip.

Above experiment results show that the dynamic performance of digital secondary equipment can be tested effectively by the new testing technology. 


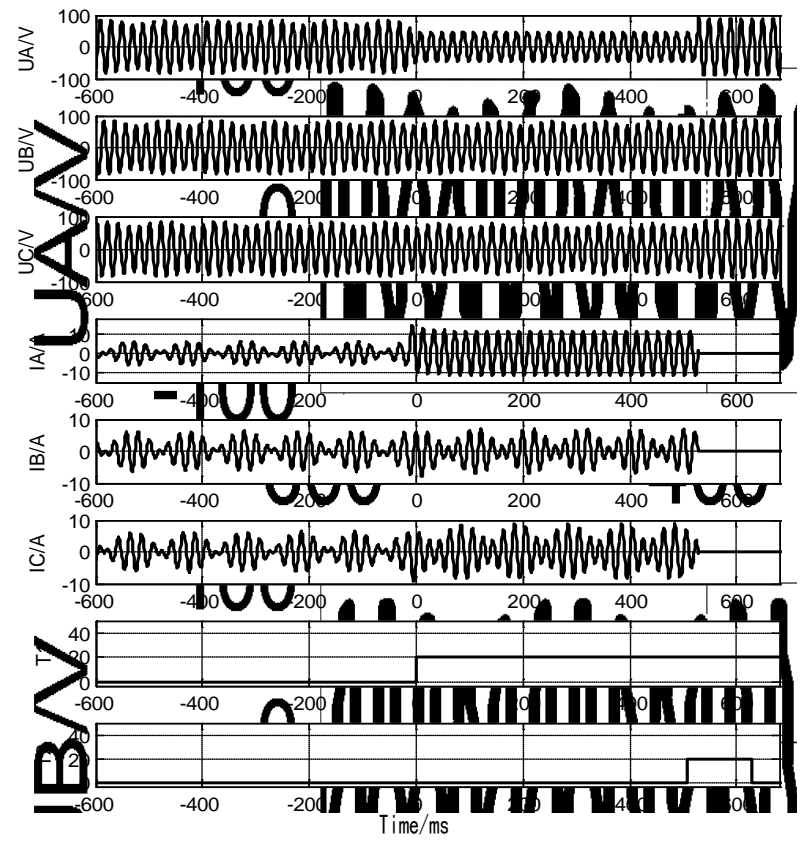

Figure 8. Transformer out-area fault during power grid oscillation.

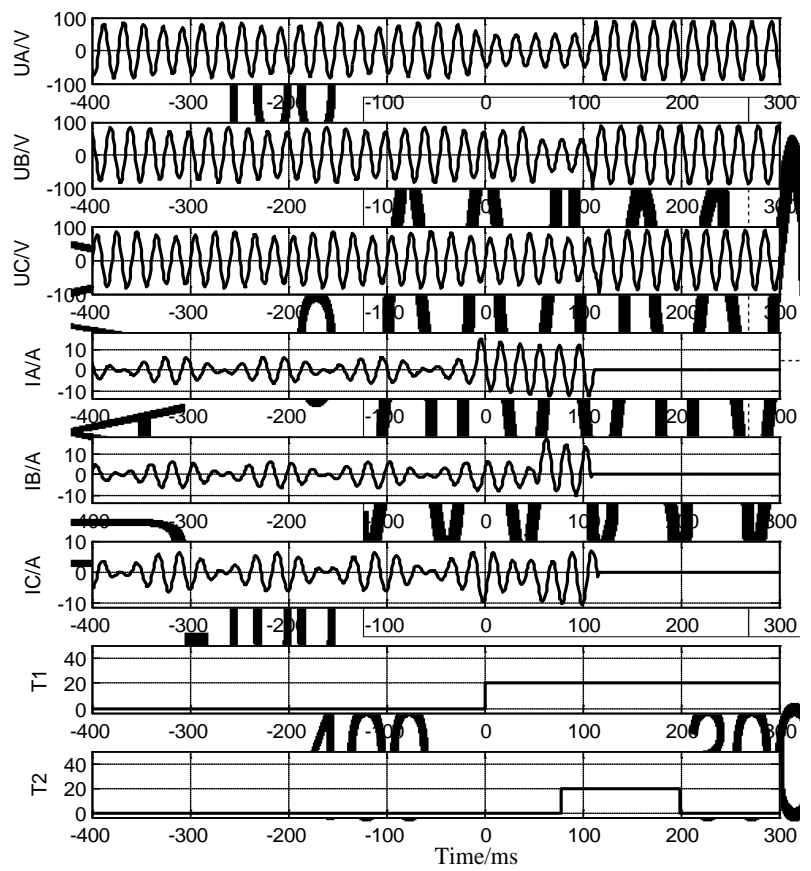

Figure 9. Transitional fault during power grid oscillation.

\section{Acknowledgements}

According to the dynamic performance test requirements of secondary equipment in substation, and considering the features of smart substation secondary equipment such as digitization and informatization, a new field test technology of smart substation secondary equipment based on recurrence principle is proposed in the paper, and the technology is verified and applied. The application results show that the technology can test the dynamic performance of secondary equipment in substation effectively, and easy to be implemented. The new technology 
can be used to do routine maintenance and fault analysis of smart substation, so it has good application value. At present, the new technology also needs to do further studies for the application in coordination test of multiple protective device.

\section{References}

[1] Ye, Y.B. and Chen, S. (2010) Analysis of Successive Trips of Transformer Differential Relay and Bus Differential Relay. Electric Power Automation Equipment, 30, 146-148.

[2] Overman T M, Sackman R W. (2010) High Assurance Smart Grid: Smart Grid Control Systems Communications Architecture. Smart Grid Communications, IEEE International Conference, 19-24.

[3] Mao, P., Duan, Y.Q., Xu, Y., et al. (2006) Realization of Dynamic Detecting Relay in High Voltage Line Protection Devices. Automation of Electric Power Systems, 30, 86-89.

[4] Kuffel, R., Winnipeg, B.C., Giesbrecht, J., Maguire, T. and Wierckx, R.P. (1996) A Fully Digital Power System Simulator Operating in Real Time. Electrical and Computer Engineering, 733-736.

[5] Liang, Z.C., Ma, X.D., Wang, H.X., et al. (1999) Small-Sized Real-Time Digital Simulator for the Test of Protective relay. Automation of Electric Power Systems, 23, 27-30.

[6] Wang, Z., Zhang, M., Sun, L.S., et al. (2001) Research on DSP-Based Digital Test Equipment for Protection Relay by Dynamic Simulation. Electric Power Automation Equipment, 21, 8-10.

[7] Reddy, M.H., Kishor, G., Satheesh, G. and Reddy, T.B. (2012) Digital Simulation of Hybrid PWM Inverter Fed Induction Motor Using Two Inductor Boost Converter. Advances in Engineering, Science and Management (ICAESM), 361366.

[8] Minami, Y., Yamanaka, N., Imahori, T. and Tanaka, T. (2004) Substation Architecture with Protective Relays Using Digital Instrument Transformers and Digital Interface Technology. Developments in Power System Protection, 810813.

[9] Feng, J. (2011) Smart Subatation Principle and Testing Technique. China Electric Power Press, Beijing.

[10] Q/GDW 441-2010 (2012) Technical Specifications of Protection for Smart Substation. State Grid Corporation of China, Beijing.

[11] Yue, C.Y., Tian, F., Zhou, X.X., et al. (2006) Principle of Interfaces for Hybrid Simulation of Power System Electromagnetic-Electromechanical Transient Process. Power System Technology, 30, 23-27.

[12] Dube, B., Varennes, Q., Lefebvre, S., Perocheau, A. and Nakra, H.L. (1988) Comparative Hybrid and Digital Simulation Studies of the Behaviour of a Wind Generator Equipped with a Static Frequency Converter. Electrical Engineering Journal, 39-44.

[13] Zhu, X.K., Zhou, X.X., Tian, F., et al. (2011) Hybrid Electromechanical-Electromagnetic Simulation to Transient Process of Large-Scale Power Grid on the Basis of ADPSS. Power System Technology, 35, 26-31.

[14] Zhao, W.G. and Ren, X.Y. (2010) Time Synchronization with IRIG-B Code in Smart Electronic Devices. Automation of Electric Power Systems, 34, 113-115.

[15] Shi, X.H., Gao, H.L., Xiang, M.J., et al. (2011) Application of IEEE1588 Time Synchronization Protocol in Digital Substation. Electric Power Automation Equipment, 31, 132-135. 